\title{
Construindo o perfil da enfermagem*
}

Recebido em: 12/04/2012

Aceito em: 12/07/2012
Maria Helena Machado'

Ana Luiza Stiebler Vieira ${ }^{2}$ Eliane Oliveira $^{3}$

O presente artigo aborda o desenvolvimento da Pesquisa do Perfil de Enfermagem no Brasil. Focaliza as características, as tendências e a importância significativa da equipe de enfermagem na organização e no desenvolvimento das atividades de saúde, seja no âmbito hospitalar ou na rede ambulatorial.

Descritores: Enfermagem, Mercado de Trabalho, Perfil.

\section{Building the profile of nursing}

This article discusses the development of the Profile of Nursing Research in Brazil. It focuses, in particular, the size of the health sector, its trends and the significant importance of the nursing team in the organization and development of health activities in hospital environment or in outpatient network. Descriptors: Nursing, Market Work, Profile.

\section{Construir el perfil de la enfermería}

En este artículo se discute el desarrollo del Perfil de la Investigación en Enfermería en Brasil. La atención se centra, en particular, el tamaño del sector de la salud, sus tendencias y la gran importáncia del equipo de enfermería en la organización y el desarrollo de las actividades de salud en el medio hospitalario o en red ambulatorial.

Descriptores: Enfermería, Mercado de Trabajo, Perfil.

\section{INTRODUÇÃO}

\section{O emprego no macro setor saúde}

$\mathrm{O}$ setor saúde é um ramo importante na economia brasileira - representa 4,3\% dos 90,9 milhões de postos de trabalho ocupados no país -, gerando mais de $10 \%$ da massa salarial do setor formal e em torno de 3,9 milhões de postos de trabalho. Algumas tendências do setor analisadas por estudiosos ${ }^{(1-2)}$ nos anos de 1980 e 1990, mostraram uma configuração específica da área: a grande concentração geográfica e social nas grandes capitais; crescente participação das mulheres na composição da força de trabalho; a hegemonia dos médicos e da enfermagem na equipe na composição da FTS; os baixos salários no setor; o assalariamento e a perda das condições de trabalho na saúde, degradando a qualidade de vida do trabalhador da saúde. Esses estudiosos já apontavam, à época, mudanças importantes para o setor. Passados mais de doze anos, apresentamos uma situação mais confortável. Segundo dados da pesquisa desenvolvida pelo IBGE em 2009, podemos dizer que o grau de escolaridade elevou--se não só com a ampliação do quantitativo de profissionais de nível superior, mas também com as novas profissões que se inserem na equipe permanentemente. O crescimento do pessoal técnico e, mais intensamente, a qualificação da equipe de enfermagem contribuiram para esse processo. A composição dos empregos de saúde aponta para um maior equilíbrio entre os profissionais de nível superior e de nível técnico e uma redução do pessoal de nível elementar. 0 trabalho em saúde tem sido desenvolvido e ampliado atualmente por meio de equipes multiprofissionais e multidisciplinares, requerendo cada vez mais a incorporação de novos profissionais. A centralidade médica deu lugar à interdisciplinaridade, evocando profissionais a atuarem em áreas nunca imagináveis.

A equipe profissional que produz assistência à saúde é formada por médicos, enfermeiros, odontólogos, farmacêuticos, biólogos, psicólogos, nutricionistas, fisioterapeutas, terapeutas ocupacionais, além das recentes inserções de engenheiros biomédicos, economistas, sociólogos, entre outras que deverão incorporar-se à equipe em breve ${ }^{(3)}$.

Como já sabido, a feminilização é outra característica forte do setor, ou seja, a maioria da FTS é feminina, representando atualmente mais de $70 \%$ de todo o contingente - e com tendência ao crescimento para os próximos anos. Em algumas profissões, esse processo de feminilização ultrapassa a 90\%. É o caso da equipe de enfermagem que é formada quase que integralmente de mulheres. Contudo, avizinha-se um novo cenário: os dados demonstram que há uma presença crescente do contingente masculino na enfermagem, mostrando como uma tendência que veio para ficar.

Outra característica do mercado ${ }^{(4,5)}$ de trabalho em saúde é o uso intensivo e diversificado de mão de obra. Os empregos tiveram uma importante expansão na última década - passaram de 1,4 milhões em 1992 para mais de três milhões em 2009. Contudo, as modalidades de contratação, bem como as diversas

1 Socióloga. Doutora em Sociologia. Pesquisadora Titular da ENSP/FIOCRUZ e Coordenadora Geral da Pesquisa Perfil da Enfermagem no Brasil.

2 Enfermeira. Doutora em Enfermagem. Pesquisadora Titular da ENSP/FIOCRUZ e Coordenadora Geral Adjunta da Pesquisa Perfil da Enfermagem no Brasil.

3 Nutricionista. Mestre em Saúde Pública. Pesquisadora Colaboradora do Núcleo de Estudo e Pesquisa em Recursos Humanos em Saúde da ENSP e Membro da Equipe da Pesquisa Perfil da Enfermagem no Brasil.

*Este artigo é parte da pesquisa: Perfil da Enfermagem no Brasil, que está sendo desenvolvida pela ENSP/FIOCRUZ em parceria com o Cofen, ABEn e FNE e com apoio do Ministério da Saúde e da OPAS. 
jornadas de trabalho mostram a complexidade dos vínculos. $\mathrm{O}$ setor comporta uma diversidade de jornadas de trabalho que vai desde 12 horas semanais, 20, 24, 32, 40 até 44 horas.

Não tão menos importante é a presença majoritária ${ }^{(6)}$ do setor público constituindo o maior empregador do setor, mais especificamente, na esfera municipal. Contudo, não se pode desconsiderar a enorme relevância do setor privado na prestação de serviços de saúde no âmbito do SUS, através dos convênios com a rede hospitalar.

É fato que o processo ${ }^{(7)}$ de trabalho e o mundo de trabalho estão se transformando de forma significativa, alterando a modalidade de inserção dos trabalhadores, os vínculos de trabalho, a forma de remuneração e muito fortemente o modo de trabalhar, configurando-se em arenas competitivas, individualizadas em ambientes pouco saudáveis.

Mas, se não bastasse esse cenário ${ }^{(8)}$ complexo, o SUS enfrenta outro grave problema: a precarização do trabalho em áreas essenciais que deveriam estar estruturadas por profissionais qualificados e inseridos diretamente no SUS, através de concurso ou por seleção pública. No entanto, verifica-se hoje nas instituições de saúde, um considerável volume de contratações de serviços por terceirização.

Segundo pesquisa realizada ${ }^{(8)}$, o trabalho precário está associado à situação de déficit ou ausência de direitos de proteção social, à instabilidade de vínculo do ponto de vista dos trabalhadores e à condição de trabalho que cria vulnerabilidade social ${ }^{(9)}$.

Já na vertente analítica que considera a precarização das condições de trabalho com vulnerabilidade social do trabalhador, observa-se que o setor convive, rotineiramente, com profissionais que estão submetidos a dupla e tripla jornadas de trabalho exercendo suas atividades com baixos salários, com incertezas e riscos, afetando assim a qualidade de vida do trabalhador em saúde. Esse aspecto impacta negativamente os pressupostos de trabalho decente descritos pela Organização Internacional de Trabalho.

A equipe de enfermagem não ficou imune a esse processo. Ao contrário, há registros estatísticos que mostram o percentual significativo de profissionais de enfermagem em situação de precarização no trabalho no âmbito do SUS. No contexto nacional, algumas tendências já se delineiam no mercado de trabalho de enfermagem, entre as quais, o crescimento do multiemprego devido aos baixos salários, principalmente do subsetor público de saúde; o crescimento do emprego no subsetor privado; e, ainda, o surgimento de novas alternativas para a enfermagem, de inserção no mercado de trabalho em saúde.

A enfermagem é apontada como categoria crítica no âmbito dos quatro países da sub-região do Mercosul, tanto no que se refere a elementos quantitativos deficitários quanto aos elementos qualitativos presentes na definição da sua estrutura como ocupação, nos conteúdos dos seus objetos de trabalho e também no plano sócio-ideológico ${ }^{(6)}$.A profissão de enfermagem, como a medicina, faz parte daquelas profissões essenciais a qualquer sistema de saúde que pressupõe atendimento de qualidade e está alicerçada em um processo de trabalho moderno e tecnicamente aceitável em sociedades desenvolvidas. O que se quer dizer é que a enfermagem é uma profissão de utilidade pública, de valor social inquestionável.

Daí a necessidade imperiosa de conhecer de forma sistemática, ampla e detalhada essa profissão essencial ao setor saúde, que traz consigo o maior contingente técnico do conjunto da força de trabalho em saúde do Brasil: a equipe de enfermagem.

A enfermagem desponta como profissão estratégica para investigação considerando-se a problemática do segmento - constitui um significativo contingente de profissionais conformados pelas categorias de enfermeiro, técnico de enfermagem e auxiliar de enfermagem. Traçar o perfil desse contingente profissional nuclear do SUS torna-se imperativo tanto para as entidades da corporação que os representam como para a academia que formam esses profissionais e, ainda, para o governo em suas três esferas: federal, estadual e municipal, que os empregam. Contudo, a lacuna do conhecimento aprofundando sobre a FT de enfermagem, à semelhança da pesquisa dos médicos, era uma constatação que vinha sendo sentida por todos que formulam, praticam e executam políticas públicas no SUS. Há um contingente de mais de 1,4 milhões de trabalhadores da enfermagem que, se somados ao contingente médico, passam a representar em torno de $70 \%$ da Força de Trabalho em Saúde que operam no SUS. Dessa forma, torna-se imperativo conhecer a estrutura e a dinâmica do mercado de serviços de enfermagem no Brasil.

\section{Traçando o perfil da enfermagem}

Considerando que as mudanças no mundo do trabalho vêm exigindo readaptação dos profissionais ao mercado, assim como dos profissionais de saúde na reorganização do setor no país, torna-se fundamental que tenhamos um retrato amplo e mais recente de como a categoria da enfermagem vem processando sua inserção neste contexto.

Vale destacar que, historicamente, dispomos de um primeiro estudo de abrangência nacional, de 1956/1958, ou seja, o Levantamento de Recursos e Necessidades de Enfermagem, realizado pela Associação Brasileira de Enfermagem - $A B E n^{(7)}$.

$O$ segundo estudo da enfermagem brasileira ${ }^{(8)}$, realizado por iniciativa do Conselho Federal de Enfermagem - Cofen - e da Associação Brasileira de Enfermagem - ABEn -, data de 1982/1983. Portanto, para os dias atuais, não corresponde à realidade da sua situação no cenário nacional. Durante anos, esse estudo se manteve como fonte de referência imprescindível nos estudos, análises, e na compreensão da enfermagem brasileira.

Ressalta-se, ainda, o estudo de Vieira, que também constitui referência na análise da enfermagem brasileira comparada com a enfermagem dos demais países do Mercosul, no que tange recursos humanos, formação, regulação profissional e mercado de trabalho em saúde ${ }^{(9)}$.

Segundo dados do Conselho Federal de Enfermagem ${ }^{(10)}$ estavam cadastrados em março de 2010, 1.449 .583 profissionais, sendo 287.119 (19,8\%) enfermeiros, 625.863 (43,2\%) técnicos, $533.422(36,8 \%)$ auxiliares de enfermagem. A região Sudeste 
concentra o maior contingente de profissionais de enfermagem: 759.120 , ou seja, $(52,4 \%)$ da força de trabalho. Se comparadas às relações de profissionais por mil habitantes temos: Nordeste $(5,4)$, Norte $(6,1)$; Centro-Oeste $(6,5)$; Sul $(9,0)$ e Sudeste $(9,4)$.

Os dados disponibilizados pelo Cofen mostram que a equipe de enfermagem representa um elevado contingente da força de trabalho em saúde, e conhecê-la representará um passo fundamental e estratégico para as políticas públicas de gestão do trabalho e da educação no SUS. Além da importância deste estudo como referência nacional e internacional para conhecer o Perfil da Enfermagem no Brasil, espera-se que os resultados qualifiquem a oferta e a demanda dos trabalhadores; contribua para a formulação de políticas públicas tanto no setor saúde quanto no setor educação, no que se refere a aspectos da gestão, da regulação e da educação da enfermagem tendo em vista a sua inserção no Sistema Único de Saúde.

A pesquisa contribuirá também para as entidades representativas das categorias de enfermagem, no delineamento e reordenamento de políticas para o exercício profissional e para a regulação do trabalho na realidade brasileira, apontando tendências e perspectivas. Subsidiará, igualmente, o estabelecimento de uma política de formação, de trânsito profissional, de regulamentação e controle do exercício profissional no Mercosul, que prevê o livre trânsito de trabalhadores nos mercados de trabalho do Brasil, Argentina, Uruguai, Paraguai e Venezuela.

A proposta é, portanto, que de forma colaborativa entre a Fiocruz, através do Núcleo de Estudos e Pesquisas em Recursos Humanos em Saúde da Escola Nacional de Saúde Pública - ENSP, o Conselho Federal de Enfermagem - Cofen, a Associação Brasileira de Enfermagem - ABEn - e a Federação Nacional de Enfermagem - FNE-, possam oferecer o retrato atual da enfermagem, buscando conhecer e analisar o perfil das categorias de enfermagem considerando as condições de trabalho, emprego e formação, desde seus aspectos econômicos, sociais, até os aspectos éticos e políticos que envolvem as práticas desse conjunto de profissionais.

Desta forma, para o desenvolvimento da pesquisa, contará com o apoio da Secretaria de Gestão do Trabalho e da Educação em Saúde do Ministério da Saúde, da Rede de Observatório em Recursos Humanos em Saúde - ROREHS, da Organização Pan-Americana de Saúde - OPAS, do Fórum Nacional dos Auxiliares e Técnicos de Enfermagem, da Confederação Nacional dos Trabalhadores da Saúde - CNTS, da Confederação Nacional dos Trabalhadores da Seguridade Social - CNTSS, da Associação Nacional de Auxiliares e Técnicos de Enfermagem - Anaten, dos Conselhos Regionais de Enfermagem - Coren's, da Associação Brasileira de Enfermagem (estaduais) e da Federação Nacional dos Enfermeiros (entidades associadas).

O objetivo central deste estudo é o de analisar a situação atual da enfermagem no Brasil buscando conhecer a sua dinâmica no recente contexto socioeconômico e político brasileiro, traçando assim o Perfil da Enfermagem no Brasil nos seus vários aspectos. Mais especificamente, o estudo traçará o perfil dos enfermeiros, técnicos e auxiliares de enfermagem, considerando as características socioeconômicas, a formação, o desenvolvimento profissional, a inserção no mundo do trabalho e a participação sociopolítica desses profissionais; analisará a dinâmica atual do mercado de trabalho da enfermagem; apontará tendências e perspectivas para a enfermagem.

\section{O caminho metodológico}

A Pesquisa Perfil da Enfermagem no Brasil buscará caracterizar, por meio de um levantamento amostral, o contingente de enfermeiros, técnicos e auxiliares de enfermagem em atividade no país, enfocando aspectos sociodemográficos, formação profissional e acesso à informação técnico-científica, o mundo do trabalho e aspectos político-ideológicos. Para isso, se baseará nos dados de todas as unidades da federação, e posteriormente, de cada região brasileira, para então traçar o Perfil da Enfermagem no Brasil. A população de análise é constituída por todos os enfermeiros, técnicos e auxiliares de enfermagem, denominados Equipe de Enfermagem. Adotou-se como descritor do universo o cadastro desses profissionais inscritos em todos os Conselhos Regionais de Enfermagem, totalizando 1.449 .569 profissionais de enfermagem em âmbito nacional. Destaca-se, no entanto, que foram excluídos os 7.056 registros que estavam em branco, o que resultou a redução do universo para 1.442 .513 a serem pesquisados.

Para a seleção da amostra, adotou-se o método de amostragem estratificada. A população foi dividida em 54 estratos constituídos pela combinação entre as 27 unidades da federação e as duas categorias profissionais (enfermeiros $x$ técnicos e auxiliares de enfermagem). Dessa forma, o plano de amostragem permitirá estabelecer estimativas independentes para as unidades da federação segundos categorias profissionais.

A amostra é constituída pelas seguintes variáveis: sexo, idade, localização geográfica (capital/interior) e a proporção de profissionais por estados. $\mathrm{O}$ tamanho de cada amostra regional será distribuído proporcionalmente ao número de profissionais (enfermeiros, técnicos e auxiliares de enfermagem) por unidade da federação. A amostra estimada é 45 mil profissionais.

Os dados da pesquisa serão coletados através de um questionário específico enviado pelo Cofen aos sujeitos selecionados na amostra, considerando as três categorias de enfermagem: enfermeiros, técnicos e auxiliares de enfermagem. O instrumento contém questões abertas, semiabertas e fechadas com código de respostas, e está dividido em 7 blocos, a saber: bloco 1- identificação socioeconômica; bloco 2- formação profissional (enfermeiros); bloco 3- formação profissional (auxiliares e técnicos de enfermagem); bloco 4-acesso à informação técnico-científica; bloco 5- mercado de trabalho; bloco 6- satisfação no trabalho e relacionamento; bloco 7- participação sociopolítica.

O questionário e o Termo de Consentimento serão enviados pelos Correios com porte pago para devolução. O questionário poderá também ser respondido através dos sites das Instituições (Fiocruz, Cofen, ABEn e FNE), nos níveis nacional e regionais. Para acesso ao questionário on-line, o respondente antecipadamente deverá registrar o seu aceite no Termo de 
Consentimento Livre e Esclarecido. Assim, somente após o aceite ele terá o acesso ao questionário pelo site://www.ensp.fiocruz. br/perfildaenfermagem/.

O Projeto foi aprovado e autorizado pelo Comitê de Ética em Pesquisa da Escola Nacional de Saúde Pública Sérgio Arouca, da Fundação Oswaldo Cruz, para ser executado através do protocolo de pesquisa: CEP/ENSP - n. 09/10 CAAE: 000-9.0.031.000-10. A participação dos sujeitos no preenchimento do questionário será voluntária, mediante assinatura do Termo de Consentimento e na sua participação será garantido o anonimato.

Os resultados da pesquisa serão divulgados por meio de relatórios, e posteriormente serão encaminhadas cópias para as instituições parceiras e demandantes desta investigação: Conselho Federal de Enfermagem, Associação Brasileira de Enfermagem, Federação Nacional dos Enfermeiros, bem como para as instituições colaboradoras: Ministério da Saúde/Secretaria de Gestão do Trabalho e da Educação na Saúde, Organização Pan-Americana de Saúde e Fórum Nacional dos Auxiliares e Técnicos de Enfermagem. A pesquisa será amplamente divulgada pela Rede de Observatório de $\mathrm{RH}$ do Ministério da Saúde, além dos sites da FIOCRUZ e da Escola Nacional de Saúde.

Os produtos da pesquisa serão desenvolvidos em parceria com as entidades profissionais e pesquisadores com expertise na área de enfermagem. Para atingir tal objetivo, a coordenação geral da pesquisa pensou em oito grupos de trabalho que tratarão com mais profundidade as questões que afetam a profissão e que trazem graves consequências para a prestação de serviços de saúde de qualidade. $O$ trabalho da enfermagem nos Grandes Empregadores Hospitalares é considerado um GT de grande importância entre estes e, por isso, resolvemos fazer entrevistas com os enfermeiros que neles trabalham. Para isso, estamos selecionando as instituições por porte de empregos de enfermeiros, e elegendo as mais importantes dentro das 27 capitais do país. Estaremos assim considerando as peculiaridades dos estados que podem assegurar assistência através da atenção básica ou dos hospitais. De antemão sabemos que é nos hospitais que existe uma maior complexidade funcional, pela elevada busca de resolutividade. Consequentemente, os enfermeiros têm uma maior sobrecarga de trabalho, gerando altos níveis de estresse e insegurança para o exercício da profissão.

Outros temas não menos importantes que vêm afetando a prática profissional que também serão foco de estudos: falta de autonomia profissional; ambientes de trabalho desfavoráveis devido à precariedade do processo de trabalho, terceirização, regulação profissional e falta de segurança, o que tem contribuído para o aumento da violência contra enfermeiros nos locais de trabalho. Para isso foram criados os seguintes GTs: 1Conformação da Profissão de Enfermagem; 2- Regulação do Trabalho em Saúde - Enfermagem; 3- Formação e Educação Profissional; 4- Mercado de Trabalho e a Enfermagem; 5Migração, Mercosul e Integração Regional; 6- Promoção e Saúde do Trabalhador de Enfermagem; 7- Organizações Corporativas e 8- Enfermagem nos Grandes Empregadores Hospitalares.

E por fim, destacamos os produtos da pesquisa:

- Produção e impressão de seis relatórios finais para cada região: Norte, Sudeste, Nordeste, Sul, Centro-Oeste e Brasil;

- Produção e publicação de seis artigos científicos atinentes à área; - Publicação em número especial de uma revista específica para os artigos dos GTs 1, 2, 3, 4, 5, 6 e 7;

- Publicação de livro ou número especial de revista sobre o resultado do GT 8 - Enfermagem nos Grandes Empregadores Hospitalares ; - Publicação de livro contendo o Perfil da Enfermagem;

Esperamos que esta pesquisa contribua sobremaneira para a compreensão da realidade da Enfermagem no Brasil para, assim, construir políticas públicas adequadas e coerentes com a realidade e as necessidades tanto deste contingente de mais 1 milhão e 400 mil trabalhadores da saúde como também para o Sistema Único de Saúde.

\section{Referências}

1. Donnangelo MCF. Medicina e sociedade. São Paulo: Pioneira; 1975.

2. Médici AC, Machado MH, Nogueira RP, Girardi SN. O mercado de trabalho

em saúde no Brasil - Estrutura e conjuntura (Texto de Apoio). Rio de Janeiro:

Secretaria de Desenvolvimento Educacional; 1992.

3. Machado MH. Trabalhadores da saúde e sua trajetória na reforma sanitária. In:

Lima NT, (organizador). Saúde e democracia: história e perspectivas do SUS. Rio

de Janeiro: Fiocruz; 2005.

4. Nogueira RP, Baraldi S, Rodrigues VA. Limites críticos das noçōes de

precariedade e desprecarização do trabalho na administração pública. In:

Observatório de recursos humanos no Brasil: estudos e análise. Brasília:

Ministério da Saúde; 2004

5. Vieira ALS. Os enfermeiros nos países do Mercosul: estudo comparado da (i)

mobilidade profissional no mercado de trabalho em saúde [tese]. Rio de Janeiro:
UFRJ/EEAN; 1998

6. Campos F, Brito P, Rigoli F. O campo dos recursos humanos para a saúde no

Mercosul. In: Recursos humanos em saúde no Mercosul. Rio de Janeiro: Fiocruz; 2002.

7. ABEn. Relatório final do levantamento de recursos e necessidades de

enfermagem no Brasil, 1956-1958. Brasilia: ABEn; 1980.

8. COFEN. O exercício da enfermagem brasileira nas instituiçōes de saúde do

Brasil: 1982/1883. Rio de Janeiro: COFEN/ABEn; [s.d.].

9. Vieira ALS. Os enfermeiros no Mercosul: recursos humanos, regulação e

formação profissional comparada. Relatório final [cd]. Rio de Janeiro: Rede

Observatório de Recursos Humanos em Saúde; 2006.

10. COFEN. Análise de dados dos profissionais de enfermagem existentes nos

Conselhos Regionais. Relatório de pesquisa. Brasilia: COFEN; 2011. 